\title{
COMMUTATIVE EXTENSIONS BY CANONICAL MODULES ARE GORENSTEIN RINGS
}

\author{
ROBERT FOSSUM ${ }^{1}$
}

\begin{abstract}
Reiten has demonstrated that the trivial Hochschild extension of a Cohen-Macaulay local ring by a canonical module is a Gorenstein local ring. Here it is proved that any commutative extension of a Cohen-Macaulay local ring by a canonical module is a Gorenstein ring. Also Gorenstein extensions of a local CohenMacaulay ring by a module are studied.
\end{abstract}

Introduction. Suppose $A$ is a commutative ring and that $M$ is an $A$-module. A commutative extension of $A$ by $M$ is an exact sequence of abelian groups

$$
0 \longrightarrow M \stackrel{i}{\longrightarrow} E \stackrel{\pi}{\longrightarrow} A \longrightarrow 0,
$$

where $E$ is a commutative ring, the map $\pi$ is a ring homomorphism and the $A$-module structure on $M$ is related to $(E, i, \pi)$ by the equations

$$
e i(x)=i(\pi(e) x), \quad \text { for all } e \in E \text { and all } x \in M \text {. }
$$

The $i$ identifies $M$ with an ideal of square zero in $E$. (On the other hand if $\mathfrak{J}$ is an ideal of square zero in $E$, then $\mathfrak{I}$ is an $E / \mathfrak{J}$-module and $0 \rightarrow \mathfrak{J} \rightarrow$ $E \rightarrow E / \mathfrak{J} \rightarrow 0$ is an extension of $E / \mathfrak{I}$ by $\mathfrak{J}$.)

The trivial extension of $A$ by $M$ is the exact sequence

$$
0 \longrightarrow M \stackrel{i}{\longrightarrow} M \times A \stackrel{\pi}{\longrightarrow} A \longrightarrow 0
$$

where $i$ is the first coordinate map, where $\pi$ is the second projection and where $M \times A$ is a ring whose underlying additive structure is the direct sum of abelian groups and whose multiplication is given elementwise by $(m, a)\left(m^{\prime}, a^{\prime}\right)=\left(m a^{\prime}+m^{\prime} a, a a^{\prime}\right)$ for all $m, m^{\prime} \in M$ and all $a, a^{\prime} \in A$. This extension is denoted by $A \ltimes M$.

Now suppose $A$ is a commutative noetherian local ring with maximal ideal $m$. An $A$-module of finite type $M$ is a canonical module if it has the

Received by the editors September 30, 1972.

AMS (MOS) subject classifications (1970). Primary $13 \mathrm{H} 10$.

Key words and phrases. Noetherian, Gorenstein, Cohen-Macaulay, local ring, canonical module, extension.

1 This research was partially supported by the NSF. I am grateful for many stimulating conversations with my colleague, P. A. Griffith. 
three properties:

(i) The natural homomorphism $A \rightarrow \operatorname{End}_{A}(M)$ is a bijection.

(ii) The groups $\operatorname{Ext}_{A}^{i}(M, M)=0$ for all $i>0$.

(iii) The injective dimension $\operatorname{id}_{A} M<\infty$. If $B$ is a Gorenstein local ring (see Bass [1]) and if $B \rightarrow A$ is a surjection then $A$ is a Cohen-Macaulay ring if and only if $\operatorname{Ext}_{B}^{i}(A, B)=0$ for $i \neq \operatorname{dim} B-\operatorname{dim} A$. If $A$ is CohenMacaulay and if $d=\operatorname{dim} B-\operatorname{dim} A$, then $\operatorname{Ext}_{B}^{d}(A, B)$ is a canonical $A$ module (see Grothendieck [5] and Sharp [8]). Furthermore $A$ is Gorenstein if and only if $A \cong \operatorname{Ext}_{B}^{d}(A, B)$. As a converse to this result Reiten [7] shows that if the Cohen-Macaulay local ring $A$ has a canonical module $M$, then the trivial extension $A \times M$ is a Gorenstein ring. A more precise statement is found in Fossum, Griffith and Reiten [3]: The ring $A \ltimes M$ is a Gorenstein ring if and only if the $A$-module $M$ is a canonical module.

For further properties of canonical modules we refer to Sharp [8], Herzog and Kunz [6], Foxby [4] and Fossum, Griffith and Reiten [3] although the only results which are really needed are:

(a) If $A$ has a canonical module $M$, then $A$ is Cohen-Macaulay.

(b) If $M$ is a canonical $A$-module and if $a \in A$, then $a$ is regular on $M$ if and only if it is regular on $A$.

(c) If $M$ is a canonical $A$-module and if $a$ is a regular element in $A$, then $M / a M$ is a canonical $A / a A$-module. Also we note that when $B \rightarrow A$ is a surjective ring homomorphism, when $B$ is Gorenstein and when $A$ is Cohen-Macaulay then the spectral sequence (Cartan and Eilenberg [2]) with $E_{2}^{p q}=\operatorname{Ext}_{A}^{p}\left(X, \operatorname{Ext}_{B}^{q}(A, B)\right)$ and abutment $\operatorname{Ext}_{B}^{n}(X, B)$ degenerates to natural isomorphisms $\operatorname{Ext}_{A}^{p}\left(X, \operatorname{Ext}_{B}^{d}(A, B)\right) \cong \operatorname{Ext}_{B}^{p+d}(X, B)$ for all $A$-modules $X$, for all integers $p \geqq 0$ and for $d=\operatorname{dim} B-\operatorname{dim} A$.

(The term canonical module seems to have been introduced by Herzog and Kunz [6]. A more geometric term is module of dualizing differentials, which is very suggestive terminology but also rather cumbersome. Sharp [8] uses the term Gorenstein module of rank one.)

Arbitrary extensions. The main result of this paper is the generalization of Reiten's result.

THEOREM. Suppose $A$ is a local noetherian ring and $M$ is a canonical $A$-module. If $0 \rightarrow M \rightarrow^{i} E \rightarrow^{\pi} A \rightarrow 0$ is a commutative extension of $A$ by $M$, then $E$ is a Gorenstein ring.

The proof is based on two lemmas. The first allows a reduction to the Artin case. The second handles the Artin case.

Lemma 1. Suppose $A, M$ and $E$ are as in the statement of the theorem. An element $e$ in $E$ is regular if and only if $\pi(e)$ is regular in $A$. 
Proof. Since $M$ is a canonical module, an element $\pi(e)$ is regular in $A$ if and only if it is regular on $M$. If $e \in E$ and if $e$ is regular then $e$ is regular on $i(M)$. But the restriction of $e$ to $i(M)$ is the action of $\pi(e)$ on $M$. Hence $\pi(e)$ is regular in $A$.

Suppose, on the other hand, that $\pi(e)$ is regular in $A$. If $e \cdot x=0$ for some $x \in E$, then $\pi(e) \cdot \pi(x)=0$. Hence $\pi(x)=0$, since $\pi(e)$ is regular in $A$. There is then an element $m \in M$ such that $x=i(m)$. Then $e x=i(\pi(e) m)$. Since $\pi(e)$ is regular on $M$ and since $i$ is an injection, the element $m=0$. Hence $x=0$. So $e$ is regular in $E$. Q.E.D.

Lemma 2. Suppose $A$ is an Artin ring, that $M$ is an A-module with $\operatorname{Ann}_{A} M=(0)$ and that $E$ is an extension of $A$ by $M$. Then $i(\operatorname{Socle}(M))=$ $\operatorname{Socle}(E)$.

Proof. Let $n$ be the radical of $E$ and $m$ the radical of $A$ (so that $\mathfrak{m}=\pi(\mathfrak{n}))$. Now $e \in \operatorname{Socle}(E)$ if and only if $n e=(0)$. If $n e=(0)$, then $i(M) e=(0)$. But $i(M) e=i(M \pi(e))$. Hence $r e=(0)$ implies $M \pi(e)=(0)$. But $\mathrm{Ann}_{A} M=(0)$ implies that $\pi(e)=0$. Hence $e \in \operatorname{Socle}(E)$ implies $e \in i(M)$, say $e=i(m)$ for some $m \in M$. Now $n \cdot i(m)=i(m \cdot m)$ so $m \cdot m=(0)$. Hence $m \in \operatorname{Socle}(M)$ and then $e \in i(\operatorname{Socle}(M))$. Clearly $i(\operatorname{Socle}(M)) \subseteq \operatorname{Socle}(E)$. Q.E.D.

Now these two lemmas are used to prove the theorem. The proof is by induction on $\operatorname{dim} A$. We know that $\operatorname{dim} A=\operatorname{dim} E$ since $i(M)$ is nilpotent. By Lemma 1 we can conclude that depth $A=\operatorname{depth} E$. For suppose $e$ is a regular nonunit in $E$. Then $\pi(e)$ is a regular nonunit in $A$. Multiplication by $e$ induces the commutative diagram with exact rows and columns

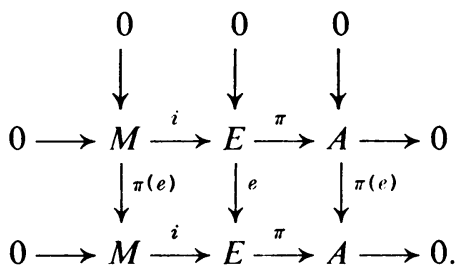

We conclude that the sequence

$$
0 \longrightarrow M / \pi(e) M \stackrel{i^{\prime}}{\longrightarrow} E / e E \stackrel{\pi^{\prime}}{\longrightarrow} A / \pi(e) A \longrightarrow 0
$$

is exact and is an extension of the local ring $A / \pi(e) A$ by the canonical module $M / \pi(e) M$. Now depth $E=1+\operatorname{depth} E / e E$. By induction depth $E / e E=\operatorname{depth} A / \pi(e) A=-1+$ depth $A$. Hence depth $E=\operatorname{depth} A$.

But we can also use this reduction to show that $E$ is Gorenstein. For $E$ is Gorenstein if and only if $E / e E$ is Gorenstein.

If $\operatorname{dim} A=0$, then the canonical module $M \cong E(A / \mathrm{m})$, the injective 
envelope of the residue class field of $A$. Hence $\mathrm{Ann}_{A} M=(0)$ since $A \cong$ End $_{A}(M)$ and $\operatorname{Socle}(M) \cong A / \mathrm{m}$. By Lemma 2 we get $\operatorname{Socle}(E) \cong E / \mathfrak{n} \cong A / \mathrm{m}$. Hence $E$ has a simple socle and is therefore Gorenstein (Bass [1]).

Suppose, for inductive purposes, that $E^{\prime}$ is Gorenstein for $\operatorname{dim} E^{\prime}<\operatorname{dim} E$ whenever $E^{\prime}$ is an extension of a local ring by a canonical module. Then we conclude that $E$ is Gorenstein since $E / e E$ is Gorenstein. Q.E.D.

Gorenstein extensions. Now our attention is directed to the converse problem. Suppose $E$ is an extension of $A$ by $M$, that $E$ is Gorenstein and that $A$ is Cohen-Macaulay. What is the relationship between $M$ and the canonical module $\operatorname{Hom}_{E}(A, E)$ of $A$ ? (Note that there are Gorenstein extensions of non-Cohen-Macaulay rings. If $k$ is a field, $X$ and $Y$ indeterminates, then $E=k[[X, Y]] /\left(X^{2}\right)$ is Gorenstein. The ideal generated by the image $X Y$ is nilpotent. But $k[[X, Y]] /\left(X^{2}, X Y\right)$ is not Cohen-Macaulay.)

Suppose $E$ is a Gorenstein local ring and $\mathfrak{I}$ is an ideal of square zero. Let $A=E / \mathfrak{I}$. We assume that $A$ is Cohen-Macaulay.

Let $B$ be a Gorenstein local ring with $\operatorname{dim} B>0$. Let $t$ be a regular element in $B$. Then $B / t^{n} B$ is Gorenstein for all $n \geqq 1$. Let $E=B / t^{n} B$ and let $\mathfrak{J}=$ $t^{n-1} B / t^{n} B$. Then $E / \mathfrak{J}=B / t^{n-1} B$ and $\mathfrak{J}^{2}=(0)$. Now $\mathfrak{J}$ is a canonical $E / \mathfrak{J}$-ideal if and only if $n=2$. Hence it is not the case that $\mathfrak{J}$ is the canonical module.

Let $\Omega=\operatorname{Hom}_{E}(A, E)$ be the canonical module. Then $\Omega \cong\{e \in E: e \mathfrak{I}=$ (0) $\}$. Since $\mathfrak{J}^{2}=(0)$, we have $\mathfrak{I} \cong \Omega$. We get the commutative diagram with exact rows and columns

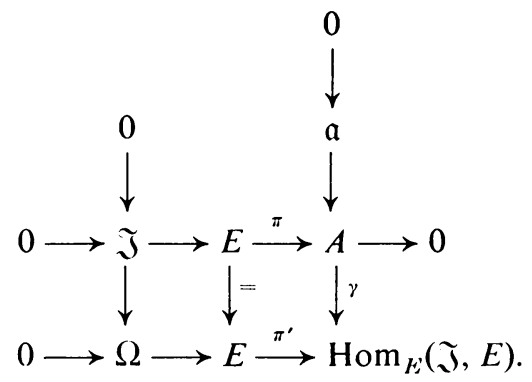

The homomorphism $\gamma: A \rightarrow \operatorname{Hom}_{E}(\mathfrak{J}, E)$ is the composition $A \rightarrow$ $\operatorname{Hom}_{E}(\mathfrak{I}, \mathfrak{I}) \rightarrow \operatorname{Hom}_{E}(\mathfrak{I}, E)$. Thus Ker $\gamma=\operatorname{Ann}_{A} \mathfrak{I}$.

Lemma 3. The natural map $A \rightarrow \operatorname{Hom}_{E}(\mathfrak{J}, \mathfrak{I})$ is a surjection and $\operatorname{Hom}_{E}(\mathfrak{J}, \mathfrak{J}) \rightarrow \operatorname{Hom}_{H}(\mathfrak{J}, E)$ is a bijection. Thus $\operatorname{Hom}_{E}(\mathfrak{I}, \mathfrak{I})$ is identified with both $A / \mathrm{Ann}_{A}$ I and $E / \Omega$.

Proof. Since $\mathfrak{J}^{2}=(0)$ and since $A$ is Cohen-Macaulay, the group $\operatorname{Ext}_{E}^{i}(A, E)=(0)$ for $i>0$. Hence $\pi^{\prime}$ is a surjection. Thus $\gamma$ is also a surjection. Since $\operatorname{Hom}_{E}(\mathfrak{J}, \mathfrak{J}) \rightarrow \operatorname{Hom}_{E}(\mathfrak{J}, E)$ is an injection, the statements of the lemma follow. Q.E.D. 
We identify $\operatorname{Coker}(\mathfrak{J} \rightarrow \Omega)$ with $a$, the annihilator of $\mathfrak{J}$. The ring $\operatorname{Hom}_{E}(\mathfrak{J}, \mathfrak{J}) \cong \operatorname{Hom}_{A}(\mathfrak{J}, \mathfrak{I})$ and is denoted by $A^{\prime}$. Then the diagram can be displayed as

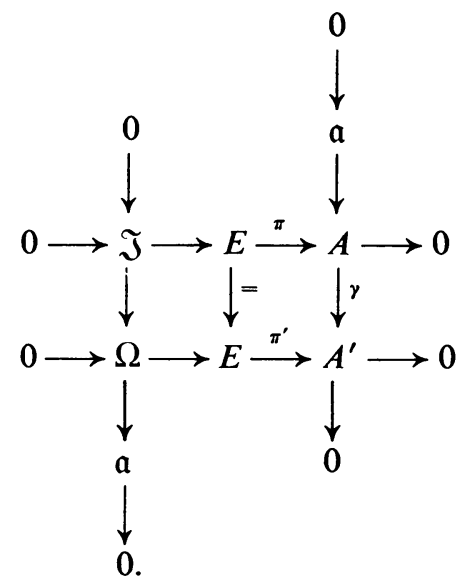

Lemma 4. The ring $A^{\prime}$ is Cohen-Macaulay and $\operatorname{dim} A=\operatorname{dim} A^{\prime}$.

Proof. It is enough to prove that $\operatorname{Ext}_{E}^{i}\left(A^{\prime}, E\right) \cong(0)$ for $i>0$. The exact sequence $0 \rightarrow \Omega \rightarrow E \rightarrow A^{\prime} \rightarrow 0$ gives rise to the exact sequence

$$
0 \rightarrow \operatorname{Hom}_{E}\left(A^{\prime}, E\right) \rightarrow E \rightarrow \operatorname{Hom}_{E}(\Omega, E) \rightarrow \operatorname{Ext}_{E}^{1}\left(A^{\prime}, E\right) \rightarrow 0
$$

and the isomorphisms $\operatorname{Ext}_{E}^{i}(\Omega, E) \cong \operatorname{Ext}_{E}^{i+1}\left(A^{\prime}, E\right)$ for $i>0$. Since $A$ is Cohen-Macaulay we get natural isomorphisms $\operatorname{Ext}_{E}^{i}(\Omega, E) \cong \operatorname{Ext}_{A}^{i}(\Omega, \Omega)$ for all $i$. Hence $\operatorname{Ext}_{E}^{i}(\Omega, E)=0$ for all $i>0$ while

$$
\operatorname{Hom}_{E}(\Omega, E) \cong \operatorname{Hom}_{A}(\Omega, \Omega) \cong A,
$$

since $\Omega$ is a canonical $A$-module. (This follows from the remarks in the introduction.) Thus $E \rightarrow \operatorname{Hom}_{E}(\Omega, E)$ is just $\pi: E \rightarrow A$ which is a surjection. So $\operatorname{Ext}_{E}^{i}\left(A^{\prime}, E\right)=(0)$ for all $i>0$ while the canonical $A^{\prime}$-module is $\operatorname{Ker} \pi=\mathfrak{J} \cong \operatorname{Hom}_{E}\left(A^{\prime}, E\right) \cong\{e \in E: e \Omega=0\}$. Q.E.D.

We record several other consequences. We retain the hypotheses and notation.

Proposition. (a) The sequences

$$
0 \longrightarrow \mathfrak{J} \longrightarrow E \stackrel{\pi}{\longrightarrow} A \longrightarrow 0 \text { and } 0 \longrightarrow \Omega \longrightarrow E \stackrel{x^{\prime}}{\longrightarrow} A^{\prime} \longrightarrow 0
$$

are $\operatorname{Hom}_{E}(-, E)$ dual.

(b) The sequences $0 \rightarrow \mathfrak{a} \rightarrow A \rightarrow A^{\prime} \rightarrow 0$ and $0 \rightarrow \mathfrak{I} \rightarrow \Omega \rightarrow \mathfrak{a} \rightarrow 0$ are $\operatorname{Hom}_{A}(-, \Omega)$ (which is $\left.\operatorname{Hom}_{E}(-, E)\right)$ dual.

(c) The group $\operatorname{Ext}_{W}^{i}(\mathfrak{a}, E)=(0)$ for all $i>0$. 
(d) The A-module $\mathfrak{J}$ is a canonical $A^{\prime}$-module while $\Omega$ is a canonical $A$-module. Thus, in particular, the square zero ideal $\mathfrak{I}$ is a canonical Amodule if and only if $\mathrm{Ann}_{A} \mathfrak{J}=(0)$.

(e) We have the following natural isomorphisms:

$$
\begin{aligned}
A & =\operatorname{Hom}_{A}(\Omega, \Omega), \\
A^{\prime} & =\operatorname{Hom}_{A}(\mathfrak{J}, \mathfrak{I}) \cong \operatorname{Hom}_{E}(\mathfrak{I}, E) \cong \operatorname{Hom}_{A}(\mathfrak{I}, \Omega), \\
\mathfrak{J} & =\operatorname{Hom}_{A}\left(A^{\prime}, \Omega\right) \cong \operatorname{Hom}_{E}\left(A^{\prime}, E\right), \text { and } \\
\Omega & =\operatorname{Hom}_{A}(A, \Omega) \cong \operatorname{Hom}_{E}(A, E) . \quad \text { Q.E.D. }
\end{aligned}
$$

Note that $\Omega$ is a nilpotent ideal in $E$ which has square zero if and only if $\Omega=\mathfrak{I}$.

Final remarks. A result which is implicit in this article, and which follows immediately is a slight generalization of the Grothendieck-BassSharp result about Cohen-Macaulay factor rings of Gorenstein rings. We state it here for possible future reference.

Proposition. Suppose A is a local Cohen-Macaulay ring with a canonical module $\Omega$. The factor ring $A^{\prime}$ of $A$ is Cohen-Macaulay if and only if $\operatorname{Ext}_{A}^{i}\left(A^{\prime}, \Omega\right)=(0)$ for all $i \neq \operatorname{dim} A-\operatorname{dim} A^{\prime}$. If $A^{\prime}$ is Cohen-Macaulay, then it has a canonical module which is just the nonzero Ext group. Q.E.D.

It should be mentioned that a nontrivial extension has been exhibited at the beginning of this section. Others will arise from symmetric 2-cocycles $f: A \times A \rightarrow \Omega$ which are not coboundaries (if they exist).

\section{REFERENCES}

1. H. Bass, On the ubiquity of Gorenstein rings, Math. Z. 82 (1963), 8-28. MR 27 \#3669.

2. H. Cartan and S. Eilenberg, Homological algebra, Princeton Univ. Press, Princeton, N.J., 1956. MR 17, 1040.

3. R. Fossum, P. Griffith and I. Reiten, The homological algebra of trivial extensions with applications to ring theory (to appear).

4. H.-B. Foxby, Gorenstein modules and related modules, Math. Scand. 31 (1972), 267-284.

5. A. Grothendieck, Local cohomology, Lecture Notes in Math., no. 41, SpringerVerlag, Berlin and New York, 1967. MR 37 \#219.

6. J. Herzog and E. Kunz, Der kanonische Modul eines Cohen-Macaulay-Rings, Lecture Notes in Math., no. 238, Springer-Verlag, Berlin and New York, 1971.

7. I. Reiten, The converse to a theorem of Sharp on Gorenstein modules, Proc. Amer. Math. Soc. 32 (1972), 417-420.

8. R. Y. Sharp, Gorenstein modules, Math. Z. 115 (1970), 117-139. MR 41 \#8401.

Matematisk Institut, Universitetsparken, Ny Munkegade, 8000 aarhus C, DENMARK

Current address: Department of Mathematics, University of Illinois, Urbana, Illinois 61801 